psychobiological model assessed by the temperament and character inventory (TCI). We aimed to determine how individuals with bipolar disorder differ from individuals with major depression (MDD) and unaffected relatives of patients with bipolar disorder using the TCI.

Methods: Data were collected from 277 adults recruited in Christchurch, New Zealand, as part of the South Island Bipolar Study. The 240-item TCI questionnaire was used to determine personality profile, and the diagnostic interview for genetic studies was used to establish four groupings, bipolar disorder type1 (BP1), bipolar disorder type-2 (BP2), MDD and nonaffected relatives. Differences identified with an ANOVA were corrected for current mood state by the inclusion of the Beck Depression Inventory as a covariate in a univariate ANOVA.

Results: Total harm avoidance was higher and selfdirectedness was lower than controls in all mood disorder groups but not different between them. Total self-transcendence was higher in BP1 than MDD and unaffected relatives. Correction for current mood influenced the significance of the differences between groups.

Conclusions: The TCI has identified commonalities and differences between bipolar disorder and MDD. Higher harm avoidance and lower self-directedness may reflect personality markers of mood disorders, whereas high self-transcendence appears to be specific to bipolar disorder. Self-transcendence may prove to be a useful measure for the investigation of the genetic basis of bipolar disorder.

\section{Orosomucoid influences both antidepressant tolerance and response}

\section{J Harley, R Roberts, P Joyce, R Mulder, S Luty, C Frampton, M Kennedy}

Christchurch School of Medicine and Health Sciences, University of Otago, New Zealand

Background: Orosomucoid, an acute-phase reactant (APR), carries basic drugs including antidepressants. Elevated levels have been reported in depressed patients. It has yet to be established whether concentration influences antidepressant response. Its gene, $O R M 1$, is polymorphic and has three common codominant alleles ORM*F1, *F2 and ${ }^{*} \mathrm{~S}$. The variants have differing pharmacokinetic properties that potentially alter plasma profile and blood-brain barrier transport of antidepressants influencing tolerance and efficacy.

Methods: A total of 157 out-patients in a trial of fluoxetine vs. nortriptyline were genotyped for the ORM1 variants. Plasma concentrations of APRs were also measured. Outcomes were tolerance and response.
Tolerance was defined as the completion of a 6-week trial and response as an improvement of greater than $60 \%$ on the Montgomery-Asperg Depression Rating Scale at 6 weeks. Groups were compared using one-way ANOVA and chi-squared tests. Outcome predictions were performed using binomial logistic regression.

Results: Individuals with an ORM1*F allele were more likely to tolerate antidepressants [odds ratio $(\mathrm{OR})=4.707,95 \%$ confidence interval (CI) 1.769 $12.527, P=0.002$ ). Higher orosomucoid concentrations were found in antidepressant nonresponders (91.4\% vs. $79.1 \%$, F-stat $6.071, P=0.015)$. For every $1 \%$ increase in orosomucoid, the odds of response were decreased $(\mathrm{OR}=0.984,95 \%$ CI 0.971-0.997, $P=0.018)$.

Conclusions: The two effects of orosomucoid - polymorphism affecting tolerability and concentration affecting efficacy - emphasize its importance in the handling of antidepressants.

\section{Development of an antenatal intervention package for women with serious mental illness}

\author{
Y Hauck', D Rock ${ }^{1,2}$, T Jackiewicz ${ }^{3}$, A Jablensky ${ }^{1}$
}

${ }^{1}$ Centre for Clinical Research in Neuropsychiatry (CCRN); ${ }^{2}$ North Metropolitan Area Health Service; and ${ }^{3}$ Telethon Institute for Child Health Research (TICHR), Perth, Australia

Background: Women with a serious mental illness (SMI) are at high risk for pregnancy and birth complications that increase childhood neurodevelopmental risks (Jablensky et al. 2005). Lifestyle hazards, such as smoking, use of street drugs and poor nutrition, as well as failure to access antenatal care, have been implicated in this increased risk. However, evidence from the relevant literature suggests that during pregnancy, many women with SMI continue their contact with mental health services.

Purpose: With a primary prevention focus, the purpose of this project was to develop an antenatal intervention package for community mental health clinicians as a strategy to reduce risks and improve the reproductive health outcomes for women with SMI.

Methods: A qualitative exploratory study involved consultation with key stakeholders, an environmental scan to determine current service delivery issues and a review of the literature. Individual and focus group interviews were undertaken with community mental health clinicians, consumers and midwives.

Results: An antenatal programme of care, including clinical guidelines and information regarding antenatal care and support services to facilitate the role of 
community mental health clinicians as advocates for their pregnant clients, has been developed. Additional outcomes include an education package to up-skill clinicians in using the resource, a surveillance system to identify at-risk cases and an information system to enable evaluation of the impact on obstetric and child health outcomes.

Conclusion: This primary preventive intervention has the potential to significantly improve obstetric and neonatal outcomes for this high-risk cohort.

\section{Participatory action research: researching with disenfranchised populations}

\section{W Hill, T Koch}

University of Newcastle, Newcastle, Australia

Background: The aim of this study was to position participatory action research (PAR) as a vital, dynamic and relevant approach that can be used when researching disenfranchised populations. PAR enables people to contribute their ideas, and plan and partake in effective action to improve their lives.

Method: We have developed a chronic illness research program that has been guided by participatory principles. Research to date includes the following: the needs of people who were learning to live with human immunodeficiency virus (HIV) and the intrusion of fatigue, learning alongside older people with asthma, people (homeless men living with schizophrenia) who experienced incontinence, Aboriginal elders who wanted to develop strategies to bring their plight of the high incidence of diabetes to the attention of their community, and women who were homeless and had been sexually violated as children. In addition, PAR as a legitimate methodology, the literature identified the following disenfranchised groups: persons with chronic illnesses such as HIV/acquired immunodeficiency syndrome and depression $(n=26)$, psychiatric diagnoses (eg clinical depression, schizophrenia) $(n=15)$, survivors of abuse $(n=6)$, alcohol misuse $(n=2)$, illicit drug use $(n=4)$, prescription drugs use $(n=4)$, prison populations $(n=2)$ and people with organic brain disorders $(n=5)$.

Results/Conclusions: While we have used action research approaches to research with people to explore disruptive events and develop ways they can transition through the event to create a sense of continuity in their lives, the literature has established that PAR is now a well-established methodological means for engaging with disempowered and/or marginalized populations into their own health management.

\section{Validation of a pencil-and-paper measure for depression in the cardiac population: the DMI-10 and DMI-18}

\section{T Hilton, G Parker}

The Black Dog Institute, Sydney, Australia

Background: Two issues arise in relation to depression in the cardiac population. The first is in relation to clinical practice, although depression is now recognized as a risk factor for increased morbidity and mortality in those with acute coronary syndrome (ACS) and, to a lesser degree, heart failure (HF). It remains underdiagnosed and undertreated in clinical practice. The second issue is a measurement issue: much of the research on depression in the cardiac population relies on measurement of depression symptoms with psychiatric rating scales and applies cut-offs for these scales that were predetermined for use in psychiatric rather than medical setting. This may lead to measurement errors as many of these scales include somatic symptoms. A well-validated screening and measurement tool specifically validated for use in this population may speak to both these issues, aiding detection and treatment of this important risk factor, as well as measuring it more accurately for research purposes.

Methods: A total of 322 patients with ACS or HF completed the DMI measures, psychosocial questionnaires and a semistructured clinical interview during the hospital stay.

Results: Both measures showed good psychometric properties, with high sensitivity and specificity when evaluated against clinical judgment. Cut-off points of $\geq 6$ and $\geq 14$ were determined for the DMI- 10 and DMI-14, respectively.

Conclusion: The DMI-18 and DMI-10 are appropriate for use as screening instruments in cardiac patients.

\section{Neurological soft signs in schizophrenia: using transcranial magnetic stimulation to investigate motor overflow}

\section{K Hoy ${ }^{1,2}$, N Georgiou-Karistianis ${ }^{2}$, R Laycock ${ }^{3}$, P Fitzgerald ${ }^{1,2}$}

${ }^{1}$ Alfred Psychiatry Research Centre; ${ }^{2}$ Monash University, Melbourne, Australia; and ${ }^{3}$ La Trobe University, Bundoora, Victoria, Australia

Background: Neurological soft signs (NSSs) are impairments that indicate nonspecific cerebral dysfunction. NSSs are thought to be early premorbid traits of 